\title{
Populismo y nativismo en las derechas radicales de Polonia: entre el pasado y el presente
}

Populism and Nativism in the Radical Right in Poland: Between the Past and the Present

Cómo citar este

artículo en APA:

Goldtein, A. (2021).

Populismo y nativismo

en las derechas radicales

de Polonia: entre el

pasado y el presente.

Analecta Política, 11(21),

179-200. doi: http://

dx.doi.org/10.18566/

apolit.v11n21.a01

Fecha de recepción:

28.05.2021

Fecha de aceptación:

20.07.2021

\section{ARIEL GOLDSTEIN}

Investigador Adjunto del Conicet Instituto de Estudios de América Latina y el Caribe - Argentina Universidad de Buenos Aires arielgoldstein@hotmail.com 


\section{Resumen}

La propuesta consistió en explicar el éxito de las derechas populistas radicales en Polonia desde dos perspectivas. La primera considera aspectos históricos y culturales, el desarrollo del antisemitismo y la constitución del Estado nacional luego de la Segunda Guerra Mundial. La segunda estriba en el análisis del ecosistema de derechas radicales actual, para comprender sus ideas, programas y liderazgos. El artículo se nutrió de entrevistas a académicos y del análisis de los programas de los principales partidos de la derecha populista radical en Polonia. Los conceptos de populismo y nativismo son utilizados para el análisis.

Palabras clave: Polonia, derecha radical, populismo, nativismo, partidos.

\section{Abstract}

The proposal consisted in explaining the success of the radical populist right in Poland from two perspectives. The first considers historical and cultural aspects, the development of anti-Semitism and the constitution of the national State after the Second World War. The second is the analysis of the current radical right-wing ecosystem, to understand their ideas, programs and leadership. The article was nourished by interviews with academics and an analysis of the programs of the main radical populist right parties in Poland. The concepts of populism and nativism are used for the analysis.

Keywords: Poland, radical right, populism, nativism, parties. 


\section{Introducción: derechas radicales, populismo y nativismo}

Este artículo se propone abordar la fortaleza de la derecha polaca actual desde dos perspectivas complementarias. Por un lado, los factores de cierta duración que se han hecho presentes entre finales del siglo XIX y mediados del siglo XX con el fin de la Segunda Guerra Mundial como la presencia del antisemitismo y la existencia de un Estado católico monocultural. ${ }^{1}$ Para esto, son consideradas las condiciones históricas y culturales que permitieron la afirmación de una hegemonía que hoy se despliega en distintas fuerzas políticas de la derecha radical. Por el otro, el análisis del ecosistema actual de las derechas populistas radicales, en que encontramos la competencia entre una derecha que se encuentra en el Gobierno y otra que se ha desarrollado por fuera de este. Esta idea del ecosistema se basa en la premisa de que para reconstruir la actuación de los partidos de derecha radical en los márgenes hay que también analizar a los partidos mainstream de derecha que gobiernan y forman parte de las condiciones y el contexto en el que se desarrollan los primeros. Sobre este último aspecto, tendremos en consideración a dos autores que han desarrollado su análisis sobre las características del "populismo de derecha".

Los partidos de derecha radical combinan nativismo, populismo y autoritarismo como parte de sus programas políticos. Mudde (2017, 2019) establece una distinción entre populismo y nativismo. Mientras el populismo divide a la sociedad en función de criterios morales, entre una élite corrupta y un pueblo auténtico, el nativismo divide a la sociedad en función de criterios étnicos. Lo más propio del populismo de derecha radical es el nativismo, que implica una combinación de nacionalismo y xenofobia. Es una ideología que sostiene que los Estados deben ser habitados por sus miembros nativos y que los no nativos amenazan la constitución homogénea del Estado nacional. ${ }^{2}$ La derecha radical mantiene su apego a la democracia, pero no es liberal. Gobierna sobre la base de una representación mayoritaria que pone en jaque los mecanismos de la democracia liberal. En este sentido, se diferencia de la extrema derecha, que rechaza el régimen democrático.

1 Es preciso considerar históricamente que Polonia durante muchos siglos ha sido un país multicultural y donde los judíos podían aprovechar la libertad religiosa. Así, el antisemitismo mantiene hondas raíces en la historia polaca, pero también pueden encontrarse historias de solidaridad, respeto por la diversidad y cooperación (Entrevista a Rafal Pankowski, 19-01-2021).

2 Se busca crear en última instancia una "etnocracia", una democracia en la cual la ciudadanía esté basada en la etnicidad. Se pretende recrear el "Estado monocultural", cerrar las fronteras y dar a los extranjeros la opción de la asimilación o la deportación (Mudde, 2019). 
Distintos autores han abordado la cuestión del populismo. Laclau (2012) lo ha definido como un proceso político que divide a la sociedad en dos campos antagónicos. Según el autor, se establece una cadena equivalencial de demandas que cristaliza en la figura de un líder de forma afectiva y representa identidades políticas que se definen por oposición. Esta dimensión afectiva de la política, señalada también por autores como Mouffe (2007) y Panizza (2011) muestra la importancia del populismo como una forma de hacer política que tiene relevancia tanto en Europa como en América Latina. Casullo (2019) sostiene que el populismo traza una división moral que implica "una frontera entre los 'hermanos' imaginarios y quienes no pertenecen a la comunidad y, más aún, dicen qué les sucederá a los hermanos que transgredan el mandato de parentesco" (p. 68). La autora ha formulado una distinción entre "populismo de izquierda" y "populismo de derecha", en que la principal diferencia entre ambos consiste en que el primero "pega para arriba" (corporaciones y medios) y el segundo "pega para abajo" (minorías sexuales, inmigrantes, pobres). El populismo de derecha, según la autora, "suma un fuerte énfasis en la necesidad de mantener ciertas jerarquías sociales que considera 'naturales' y una obsesión xenófoba por defender los límites de la comunidad política contra factores designados como contaminantes de la pureza del 'verdadero pueblo"' (p. 130). En estos casos, "el pueblo se imagina como algo ya orgánicamente constituido cuya autenticidad debe ser protegida de contaminaciones. El pueblo debe ser cercado y defendido antes que constituido" (p. 147).

La característica principal de lo que Mudde (2019) denomina la "cuarta ola" de las derechas radicales es su paso a la política mainstream, en particular en países de Europa Central y del Este como Hungría y Polonia. Es decir, la cuarta ola supone una radicalización del mainstream sobre los temas de "políticas de identidad". Los partidos mainstream incorporan temas tradicionales de la derecha populista radical en sus plataformas de gobierno o campaña (Mudde, 2019). Según Mudde, la cuarta ola de la extrema derecha obtuvo beneficios de tres aspectos: los ataques terroristas, la recesión de 2008 y la crisis de refugiados de $2015 .^{3}$

Metodológicamente, el trabajo se basa en una revisión de la bibliografía con respecto a los antecedentes históricos y en entrevistas a destacados académicos.

3 Se pretende hablar de aspectos que, según Mudde (2019), caracterizan a la cuarta ola de la derecha internacional. Consideramos que en países como Hungría y Polonia el rechazo a la admisión de los refugiados ha tenido peso en las derechas populistas radicales, que así encuentran un argumento de oposición a la Unión Europea (UE) y a sus políticas de migración. En este sentido, las expresiones de derecha parecen haberse articulado alrededor del rechazo a la inmigración. 
Con respecto al tiempo actual, los datos son tomados del análisis de los programas políticos, estatutos y publicaciones de los partidos de la derecha populista y nativista de Polonia. Los programas revisados y publicaciones corresponden a los principales partidos (Prawo i Sprawiedliwość, Ley Justicia [PiS] y quienes componen la alianza de partidos Konfederacja Wolność i Niepodległość, Korona, Korwin y Movimiento Nacional $\left.{ }^{4}\right)$. Estos fueron identificados como las principales fuerzas partidarias de derecha populista radical en Polonia en función de su presencia en el Sejm (Parlamento polaco).

Gdula (2018) trata de mostrar que Polonia no se ajusta tanto al patrón populista del voto por la extrema derecha como justificación de un sentimiento de frustración económica. Cuando gana Ley y Justicia en 2015, Polonia no estaba como en 2001 atravesando una crisis económica, sino en un periodo de recuperación e integración a la Unión Europea (UE). Sin embargo, el Gobierno de la Plataforma Cívica (2007-2015) había reducido en forma significativa las políticas sociales, culturales y universitarias.

Las preguntas que guiarán este trabajo son estas: ¿qué trazos comunes existen entre el pasado y el presente polaco desde el punto de vista de las derechas radicales?, ¿qué características toma el ecosistema de las fuerzas populistas de derecha radical en Polonia?, ¿cuáles son sus principales actores hoy y cómo se relacionan?, ¿cuáles son sus principales ideas? y ¿en qué medida las categorías de populismo y nativismo nos permiten explicar estos fenómenos?

\section{La tradición antisemita y el Estado católico de posguerra}

Después de la Primera Guerra Mundial fue posible para Polonia, tras 123 años, recuperar la independencia. El proceso de formación de este Estado renacido implicaba varios conflictos territoriales y sociales. Sin embargo, con el fin de aquella, los polacos se sintieron perjudicados por el tratado entre Alemania y Francia que dejaba al país vulnerable entre Alemania y la Unión de Repúblicas Socialistas Soviéticas (URSS). El país experimentó periodos de gran inestabilidad, inflación ex-

4 Konfederacja Wolność i Niepodległość es un conjunto (confederación) de varios pequeños partidos políticos, incluso KORWiN, Ruch Narodowy, Konfederacja Korony Polskiej. En total, tienen 11 sobre 460 diputados en Sejm. 
trema y la unión de los terratenientes contra los judíos y las "influencias externas". Se manifestaba un enfrentamiento de la mayoría polaca contra las minorías. El líder de la independencia polaca, Józef Piłsudski, frente a la crónica inestabilidad ejecutó un golpe de Estado con el que consolidó una senda autoritaria a principios de 1926 (Kershaw, 2015). ${ }^{5}$ El golpe de 1926 llevó el título de "Sanacja", sanación de la vida pública. Así, condujo una dictadura personal hasta 1935 tras su muerte, periodo durante el cual introdujo una nueva constitución autoritaria. Parecía simpatizar a la izquierda, en comparación con el antisemitismo y el conservadurismo de los opositores Demócratas Nacionales. Rechazó la unión entre Iglesia y Estado, y los judíos se encontraban entre los más importantes apoyos al régimen de "Sanacja" de 1926 (Pankowski, 2010).

Si bien Piłsudski sostuvo una identidad abierta multicultural, que admitía la integración de los ucranianos y los judíos, desarrolló una política autoritaria y represiva con los grupos opositores. Se destaca su histórica pertenencia de izquierda al Partido Socialista de Polonia, desempeñando un rol importante en la Revolución rusa de 1905. Sin embargo, fue luego recuperado por los grupos de la derecha polaca en su carácter autoritario. La derecha conservadora se apropió de Piłsudski, un socialista en vida, luego de su muerte. En él, conviven distintas tradiciones: socialista liberal, respetuoso de la multiculturalidad, pero también represor con la oposición (Paweł Śpiewak, comunicación personal, 25 de mayo de 2021). La fuerza de Piłsudski como "héroe nacional" obedece a que, en una nación históricamente ocupada por rusos y alemanes, su fortaleza de líder de la resistencia antisoviética y la independencia polaca lo vuelve un símbolo unificador de la nación, lo que explica que en la reivindicación de su figura se oculten sus rasgos autoritarios. Su presencia histórica fue negada durante el régimen comunista (Kotwas y Kubik, 2019), pero luego de 1989 renació, reivindicado por sus aspiraciones soberanistas.

Por otra parte, Roman Dmowski se erigió como líder de los Demócratas Nacionales contra los socialistas. Es señalado como uno de los padres fundadores del nacionalismo polaco, quienes defendían un nacionalismo estricto contra los judíos, los alemanes y los rusos. Los judíos eran el $40 \%$ de la población de Varsovia, lo que activó el antisemitismo como forma de propaganda en la construcción de una identidad política nacional (Pankowski, 2010). Dmowski, líder de

5 Josef Piłsudski era del Partido Socialista Polaco, luchó contra el zarismo y desempeñó un papel en la revolución frustrada de 1905. Buscaba la independencia de Polonia. En 1918, Piłsudski despuntó como líder de la independencia polaca y luego en 1920 lideró la facción polaca en guerra contra la Rusia soviética. 
los Demócratas Nacionales, definía étnicamente la nación, quienes rechazaban el modelo de élite de la política basado en los aristócratas polacos y, en cambio, se basaban en el modelo de política de masas para difundir el mensaje nacionalista, con especial peso en el interior del país.

Dmowski colaboró con los rusos, era parlamentario de la Duma y despotricó contra judíos y alemanes. El movimiento se fue radicalizando e inspirando en los nazis y fascistas de Italia y Alemania. Estaba obsesionado en la creencia de conspiraciones sobre los judíos y masones. Definía el catolicismo como parte de la esencia de lo polaco. Esta identificación produjo el alineamiento del clero con los Demócratas Nacionales, y lo que sucede en la actualidad puede considerarse como una repetición (Pankowski, 2010). El antisemitismo fue un factor politizador y movilizador para la clase media, que despreciaba lo que denunciaba como el "dominio de los judíos" en el comercio y los negocios. Entre 1920 y 1930, el antisemitismo se convirtió en el gran unificador del movimiento.

Este movimiento proponía la migración de los judíos y el boicot a sus negocios, que, en el este de Polonia, se redujeron el $30 \%$ entre 1932 y 1937 por estas campañas, y condujeron a su aislamiento y discriminación durante 1920-1930, lo que fue clave para su exterminio posterior durante la ocupación nazi (Pankowski, 2010).

Dmowski rechazaba la relación que existía entre los judíos y las posiciones de poder que según este habían adquirido en la economía y en la relación con la nobleza, y lleva la cuestión del antisemitismo a la política nacional (Paweł Śpiewak, comunicación personal, 25 de mayo de 2021). El libro más importante de Dmowski es Pensamientos de un polaco moderno (1903), en el cual demandaba pensar en un Estado nación donde las minorías fueran excluidas del Estado polaco y se instalara el antisemitismo como cuestión central en la política del país. ${ }^{6}$ Los movimientos nacionalistas de derecha actual en Polonia rescatan la figura de Dmowski y su visión del nacionalismo polaco como equivalente al catolicismo.

Tras la ocupación nazi y después de 1945 con el exterminio de los judíos, el cambio de fronteras después de la Segunda Guerra Mundial y grandes migraciones, Polonia se convirtió en un Estado nacional católico monocultural (Prazmowska, 2010). La guerra dejó una población homogénea en este país. Los ucranianos fueron desplazados de Polonia para constituir una comunidad ho-

6 Historiadores del Holocausto señalan que, aunque Dmowski no cometió la muerte de judíos, sí es responsable de que este haya funcionado de forma tan eficiente. La base de los Demócratas Nacionales era la clase media conservadora que no toleraba experiencias radicales como el fascismo, y por tanto, veían una solución en el "nacionalismo autoritario". 
mogénea desde el punto de vista étnico. Una gran parte fue desplazada pero al interior Polonia (p. ej., a los territorios abandonados por los alemanes). A su vez, el Gobierno soviético quiso anular una resistencia de tono tanto antinazi como anticomunista (Judt, 2006).

Luego de la Primera y Segunda Guerra Mundial, la tradición multicultural polaca es eliminada en nombre de una nación homogénea, y los ideales de una nación basada en un criterio etnorreligioso de Dmowski se convierten en realidad. Pankowski (2010) retrata que tras la crisis de 1930, en Polonia existió mucha desocupación, en especial entre los jóvenes, que se volvieron revolucionarios por esa incapacidad de integrarse en el sistema. Sin embargo, ese carácter revolucionario se produjo con un fuerte componente de antisemitismo cuando los jóvenes intelectuales polacos identificaban a los judíos como parte del problema y como sus competidores. Este mismo autor plantea que, con la guerra entre los polacos y los bolcheviques, las visiones comunistas de entreguerras quedaron impugnadas para la mayoría de la población en Polonia. Por eso, el descontento anticapitalista era nucleado por la extrema derecha junto con el antisemitismo. Esto volvería a repetirse entre 1990 y 2000 en el país. Por tanto, la configuración del moderno Estado polaco como unidad étnica nacional se produce luego de la Segunda Guerra Mundial tras la eliminación de los polacos y los ucranianos. Luego de la guerra, se produjo, en 1946, un nuevo pogromo antijudío de Kielce, que llevó a migraciones masivas de esta colectividad.

Las transformaciones de la sociedad polaca de posguerra también tienen que ver con que el exterminio de los judíos deja lugar disponible para la ocupación de esos trabajos por polacos. A partir de entonces, comienza a tener mayor lugar una cultura nacional y un proceso de homogeneización (Pankowski, 2010). El moderno Estado polaco, por tanto, está fundado sobre el Holocausto, luego de la posguerra. Por eso, el éxito de las visiones que niegan la participación polaca en los crímenes de la Segunda Guerra Mundial, como veremos más adelante. Hablar sobre los crímenes polacos es cuestionar las bases de la existencia misma de la nación polaca luego de la Segunda Guerra Mundial. Por otra parte, la historia de Polonia, un país sin Estado, la lleva a una búsqueda moralista de la "unidad nacional" y tomar los conflictos internos de la sociedad como una amenaza a su propia constitución (Szacki, 1995, citado en Bill y Stanley, 2020).

Jedrzej Giertych fue uno de los que continuó el legado del movimiento iniciado por Dmowski en libros en su exilio pos-1945 en Gran Bretaña, y codificó esta tradición en términos antisemitas, antimasones y antialemanes. Desde ese legado, se fundaron las asociaciones de la Liga de las Familias Polacas y la Juventud de 
Toda Polonia, que celebran a este autor. El legado antisemita de derecha radical de la familia Giertych fue pasado de padres a hijos, hasta llegar a Maciej y luego a Roman (Pankowski, 2010). ${ }^{7}$

Existió desde el comienzo un desprecio en Polonia por la ocupación soviética (Darden y Guzmala, 2006; Prazmowska, 2010). Pankowski (2010) destaca, sin embargo, que el régimen polaco era distinto del soviético y contaba con una base popular de apoyo, un aspecto soslayado en las interpretaciones historiográficas. Pero, por otra parte, se fue forjando una tradición de lucha anticomunista que se hizo muy relevante luego de la caída del Muro de Berlín en 1989.

Con Władysław Gomułka como secretario general del régimen, se alivió la presión estalinista y Polonia se convirtió en el país más liberal del bloque soviético (Lukowski y Zawadzki, 2019). Sin embargo, en el contexto del conflicto en Medio Oriente de la década de 1960, el régimen comunista polaco autorizó nuevas purgas antijudías. Desde el poder político, en especial el general Mieczysław Moczar desplegó una campaña de nacionalismo antisemita contra Israel. Un total de 20000 judíos intelectuales fueron presionados a abandonar el país en una censura estricta impuesta por los "partisanos" de Moczar. El periodo más profundo de esta situación de antisemitismo estatal se produjo durante 1968 (Pankowski, 2010). Este era inspirado por los líderes comunistas y formaba parte de un conflicto interno entre varias fracciones dentro del partido en el Gobierno.

Durante el régimen comunista, y con los antecedentes en la historia polaca, el antisemitismo mostró sus profundas raíces con la idea conspirativa de que los judíos ocupaban demasiados espacios de poder en la sociedad. Esto derivó en exclusiones del Gobierno comunista y las universidades hacia los judíos, así como nuevas migraciones. Debe considerarse así el componente estructural que tuvo el antisemitismo para la definición de la identidad nacional polaca moderna. La constitución de una homogeneidad étnica nacional de posguerra se estructura y define a partir de la eliminación de los judíos como la "otredad" de la nación.

7 Maciej y Roman Giertych han sido importantes dirigentes del partido de extrema derecha católico llamado la Liga de las Familias Polacas. Maciej ha sido miembro del Sejm y el Parlamento Europeo como representante polaco. Por su parte, Roman Giertych ha sido ministro de Educación del PiS y ha ocupado otras importantes funciones políticas durante el Gobierno de Kaczyński. Este último en la actualidad ha adquirido posiciones más liberales que lo han acercado al político Donald Tusk. 


\section{El populismo de derecha religiosa de Ley y Justicia}

En la década de 1980, Lech Wałęsa armó una unión de trabajadores, Solidaridad, que pedía libertad de expresión y organización sin tutela del Estado. Se destacaban corrientes contradictorias que convivían dentro de Solidaridad en su creación: catolicismo, socialismo, presencia de intelectuales opositores al régimen comunista y nacionalismo. Un hecho clave fue la movilización nacionalista católica contra el régimen soviético de los trabajadores en Gdańsk. El discurso nacionalista y la mirada nacional fue el marco desde el cual percibían el mundo los dirigentes de Solidaridad (Pankowski, 2010). Muchos de los integrantes del partido PiS participaron del movimiento de Solidaridad en las terceras o cuartas filas, como los hermanos Kaczyński. ${ }^{8}$ A finales de la década de 1980, Lech Kaczyński estaba adquiriendo importancia como colaborador de Lech Wałęsa en el Sindicato Solidaridad (Artur Domosławski, comunicación personal, 6 de agosto de 2020).

Las elecciones polacas de 1989 demostraron ser la primera ocasión para la caída del comunismo en Europa del Este. Los funcionarios comunistas de alto nivel, luego de la transición, se beneficiaron de las privatizaciones, y los trabajadores, que habían producido la caída del régimen en 1989, fueron afectados con el desempleo originado por la economía de mercado. Mientras los grupos de derecha demandaban quitar al "comunismo" de la vida pública (Lukowski y Zawadzki, 2019). La experiencia de la derecha radical debe ser entendida en el clima anticomunista reinante posterior a 1989 (Rydliński, 2018).

Una de las bases de la acción política de los hermanos Kaczyński, Lech y Jarosław, fue el cuestionamiento a las negociaciones de la Mesa Redonda ${ }^{9}$ de la transición democrática y la denuncia del "pacto liberal-comunista". PiS sostiene que la transición al capitalismo fue fallida porque benefició solo a una élite, entre la que se encontraban excomunistas. Kaczyński veía estas discusiones como un acuerdo subterráneo para excluir alternativas y para que la vieja nomenklatura mantuviera sus posiciones económicas en el nuevo orden de postransición (Pytlas, 2021; Bill y

8 Otro ejemplo es el actual primer ministro, Mateusz Morawiecki, quien perteneció a Solidaridad y a movimientos anticomunistas en su juventud. Su padre también fue un político de cierta relevancia en Solidaridad.

9 La Mesa Redonda consistió en una serie de reuniones durante 1988-1989 que mantuvieron las autoridades del Partido Comunista Polaco con los dirigentes del sindicato Solidaridad frente a las protestas en Gdansk. Estas conversaciones condujeron a la apertura electoral y la transición democrática. 
Stanley, 2020). En este sentido, el partido hace una fuerte crítica a lo que denomina el "sistema poscomunista" emergente de aquella transición, en que la nomenklatura comunista habría mantenido sus posiciones de poder e identificado a la Plataforma Cívica y Donald Tusk como expresiones de ese sistema. ${ }^{10}$

Había en quienes condujeron las políticas de transición una creencia en la racionalidad económica y política del electorado, su apertura cultural y su participación cívica como parte del "consenso liberal" pos-1989, que todas las sociedades de Europa del Este irían a seguir (Bill y Stanley, 2020).

En la elección de 2001, con la caída de Solidaridad, aparece Ley y Justicia, que reúne el voto anticomunista que hasta el momento pertenecía a este partido. En aquella elección, la Liga de las Familias Polacas, liderada por Maciej y Roman Giertych, obtuvo el $8 \%$. Andrzej Lepper, quien había armado un "partido contra los ricos", obtuvo el $10 \%$, con el voto de los castigados del poscomunismo. Ya en las elecciones de 2005 se evidenciaba una fragmentación del sistema de partidos pos-1989 por el resentimiento de los desafiliados del sistema poscomunista y el surgimiento de nuevos partidos populistas de derecha que procuraban canalizar el descontento político. En las áreas más perjudicadas por la transición de 1989, luego de votar a la izquierda poscomunista y de ver cómo se desarmaban las industrias y las granjas colectivas, se convirtieron en base de apoyo para Ley y Justicia (Rydliński, 2018).

En 2005, Lech Kaczyński ganó las elecciones presidenciales, y Jarosław las parlamentarias, y se creó la coalición de gobierno. En el primer gobierno, que transcurrió entre 2005 y 2007, la coalición se estableció entre la Liga de las Familias Polacas y el Partido Agrario liderado por Lepper, y llevó a la derecha "desde los márgenes al mainstream" (Pankowski, 2010). Mientras el Partido Agrario sostenía un discurso antiliberal en lo económico, contra el establishment y las élites, la Liga de las Familias Polacas sostenía un discurso católico de unidad nacional contra los grupos LGTB, la UE y los izquierdistas (Pytlas, 2021). La Liga de las Familias Polacas tuvo un papel importante en la naturalización de un discurso de derecha radical: "Durante sus seis años en el Parlamento polaco, la Liga de las Familias Polacas promovió una atmósfera abiertamente nacionalista, xenófoba y antieuropea, expresando rechazo hacia las actitudes sociales liberales y un ostentoso apego a la Iglesia Católica” (Rydliński, 2018, p. 20). Roman Giertych, activista de derecha

10 Programa político de Ley y Justicia (2019). 
radical de la Liga de las Familias Polacas y fundador de la organización juvenil All Polish Youth, fue nombrado como ministro de Educación durante este gobierno.

En 2007, se quebró la coalición con la derrota frente a la Plataforma Cívica de Donald Tusk. Sin embargo, Jarosław aprendió la lección de su derrota. El accidente de avión en Smolensk, Rusia, donde falleció su hermano Lech en 2010, alimentó una narrativa de la conspiración para que Kaczyński volviera como actor principal (Applebaum, 2020). Kukiz '15, un partido liderado por la estrella de rock Paweł Kukiz, fue influyente para derechizar el clima político, lo que fue funcional al avance de Ley y Justicia (Szczerbiak, 2021b). En 2015, el año de campaña electoral en Polonia, Kaczyński utilizó un lenguaje que señalaba que "los migrantes traen enfermedades, insectos" (Artur Domosławski, comunicación personal, 6 de agosto de 2020).

Ley y Justicia se funda inicialmente como un partido de la ley y el orden destinado a remover antiguos comunistas de la vida pública, con un propósito moral y regeneracionista (Ost, 2018). Aparece como una restauración autoritaria del Estado social frente a las consecuencias producidas por el neoliberalismo en sociedades poscomunistas, acostumbradas a la asistencia estatal. Los trabajadores pasaron del movimiento de izquierda antiestalinista Solidaridad a la derecha de PiS, al sentirse desamparados frente a las políticas neoliberales. La promesa nacionalista de PiS a los trabajadores precarizados es la restitución nacional y social del país que permitirá un salario digno y evitará la migración de las familias a otros países donde son tratados como ciudadanos de segunda categoría. Korolczuk y Graff (2018) definen el Gobierno de PiS como "una versión autoritaria y conservadora del estado de bienestar, con políticas pronatalistas, transferencias de efectivo a las familias y un fuerte enfoque en la familia heterosexual” (p. 813).

Ley y Justicia ha desarrollado políticas sociales para la clase media baja y baja que han reactivado la economía. Se lo señala como un caso de política social progresista con recursos nacionalistas. Los votantes piensan que las políticas cambiaron su vida cotidiana, en especial en el este subdesarrollado del país. El Gobierno ofrece a los votantes una pertenencia a la comunidad y establece una frontera entre aquellos que se encuentran adentro y afuera en su pertenencia a la nación polaca. Los primeros se autoperciben como ciudadanos patriotas y de estrictos principios morales, lo que les proporciona un sentimiento de superioridad (Gdula, 2018). Kotwas y Kubik (2019), en un sentido similar, tratan la politización de la religión como una forma de marcar los límites en Polonia respecto de quienes son parte de la comunidad y quienes no lo son. La religión opera como un criterio de división moral del mundo entre "nosotros" y "ellos", y de demarcación de las 
fronteras del mundo en oposición a los otros. Los autores observan una "intensificación interrelacionada del catolicismo excluyente y el nacionalismo asertivo que resulta en una expansión de la estructura de oportunidad discursiva para los populistas de derecha que utilizan motivos religiosos en sus ideologías" (p. 460). En la misma línea, Pankowski (2010) sostiene que el populismo en Polonia conecta con la idea de que "todos los Polacos son católicos" y que las minorías etnorreligiosas no pueden ser "verdaderamente polacas".

Esta dimensión moral del populismo, de división del mundo entre dos campos antagónicos, es señalada por Casullo (2019) y Mudde (2017, 2019) como un recurso de pertenencia y movilización política característico del populismo de derecha. En el caso de Ley y Justicia, la religión opera como un modo de definir la identidad de pertenencia al Estado monocultural católico de raíces históricas.

En el programa de 2011 de Ley y Justicia, el partido pretende rescatar una visión de "Polonia solidaria" como opuesta al "darwinismo social liberal meritocrático" (Kim, 2021). PiS denomina a su gobierno una "versión polaca del Estado de bienestar". ${ }^{11}$ Uno de los aspectos que destaca PiS es el valor de la "solidaridad" como base de la "comunidad". Ley y Justicia defiende lo que denomina "justicia social" en referencia a la doctrina social de la Iglesia (DSI), y rechaza aquello que llama "darwinismo social" producido por la "ideología liberal" que prioriza la "libertad individual" ${ }^{12}$ En este sentido, el centro de la campaña de PiS ha sido las políticas sociales y asistenciales para las familias (Bill y Stanley, 2020). Una de las consecuencias que identifican Korolczuk y Graff (2018) es la articulación entre "antigénero" y antineoliberalismo que provee de programa concreto a la derecha populista actual. Así, en su programa de 2019, PiS señala que "rechazamos los principios del neoliberalismo", y así encuentra elementos para una asociación entre una política económica de izquierda y una política cultural de derecha.

Mientras en América Latina y países de Europa como España las políticas neoliberales son asociadas con partidos y líderes de derecha como Jair Bolsonaro en Brasil, el Partido Popular y Vox en España, esta es una característica similar a la propia de los populismos clásicos latinoamericanos. ${ }^{13} \mathrm{La}$ articulación entre una

11 Programa de Ley y Justicia (2019).

12 Programa de Ley y Justicia (2019).

13 Por estas características, el Gobierno de PiS presenta similitudes con el primer peronismo desarrollado en Argentina (1946-1955) y el varguismo en Brasil (1930-1945/1951-1954). Estos regímenes propusieron un Estado social autoritario y católico, que, en su alianza con la Iglesia, busca representar la unidad nacional frente al "comunismo" y las izquierdas. Así, se evidencia en estos casos la alianza con la Iglesia, la intervención estatal y una política social generosa que incluye a los excluidos (característica del populismo y el varguismo), junto con una polí- 
política cultural conservadora y una política social generosa es una novedad en el mapa de las derechas radicales. El caso europeo más parecido en estos términos, pero sin haber formado gobierno, podría ser el del Frente Nacional en Francia. ${ }^{14}$ Esto impide señalar a Ley y Justicia como una extrema derecha, aunque puede definirse como una derecha radical: un gobierno de las mayorías que pone en tensión los fundamentos de la democracia liberal, pero no cuestiona el orden democrático, como hacen los grupos de extrema derecha.

Otra de las bases de legitimación de la derecha radical consiste en brindar una visión complaciente sobre el pasado en Polonia y difundir lo que el primer ministro Mateusz Morawiecki ha definido como "nuestra verdad sobre el pasado" (Bill y Stanley, 2020). Estos usos del pasado con fines políticos constituyen una de las bases de su legitimación. La participación polaca en el genocidio nazi es negada, y se presentan exclusivamente como "víctimas". En este sentido, el programa PiS (2019) señala que "el Estado polaco, víctima del nazismo alemán y el comunismo soviético, fue retratado como cómplice de crímenes cometidos en la Segunda Guerra Mundial. No solo rompimos con una política de silencio y vergüenza, sino que empezamos a conducir con decisión una política para dar forma a una imagen positiva de Polonia en la arena internacional”. Esta visión del Gobierno presenta a los polacos exclusivamente como víctimas de la ocupación alemana y soviética, los exime de su participación o complicidad con el Holocausto y recrea una visión complaciente de la nacionalidad polaca que omite los antecedentes históricos de antisemitismo que hemos retratado, así como lo sucedido durante el comunismo. ${ }^{15} \mathrm{La}$ última campaña enfrentó en la recta final al candidato del PiS Andrzej Duda con el alcalde de Varsovia, Rafał Trzaskowski. Mientras Kaczynski acusaba al candidato opositor de carecer de un "corazón y un alma polacos", el Gobierno acusó a través de la televisión pública a Trzaskowski de "creer en el Dios de Spinoza”, con lo cual equiparó esto con una supuesta profesión religiosa judía.

tica cultural conservadora.

14 El caso de Hungría con Orbán ha sido cuestionado por las condiciones de flexibilización y bajos salarios de los trabajadores, aunque también cuenta con asistencia estatal a determinados sectores sociales.

15 En 2018, el Gobierno polaco aprobó una ley que penalizaba a quienes usaran expresiones que relataran la complicidad de polacos con el Holocausto. Sin embargo, tras el repudio internacional cosechado, el Gobierno debió dar marcha atrás con esta ley. A su vez, determinados historiadores y académicos que han procurado mostrar este pasado han sido criticados y denominados "enemigos de Polonia" por el Gobierno polaco o medios afines. Esto ha sucedido con Jan Gross, historiador que reside en los Estados Unidos y que procuró mostrar la complicidad de los polacos en el Holocausto en su libro Vecinos, donde relataba el pogromo de Jedwabne, en 1941, "atribuido durante décadas a los nazis, pero que Gross demostró que fue perpetrado por sus vecinos católicos" (Altares, 2021). 
Según Pawel Spiewak, "el papel de la Iglesia es fundamental para el PiS, los obispos polacos y los sacerdotes del campo votan por Ley y Justicia" (comunicación personal, 15 de julio de 2020). PiS reconoce a la Iglesia un rol fundamental en la constitución de la identidad polaca, al señalar que "la Iglesia católica ha desempeñado en Polonia y desempeña un papel único, diferente al de la historia de otras naciones". ${ }^{16}$ Luego de Juan Pablo II, hay un proceso de radicalización nacionalista de derecha en la Iglesia polaca. En este contexto, la organización jurídica Ordo Iuris ha desarrollado un papel importante en Polonia en su oposición a políticas LGBT y las denuncia de lo que denominan la "ideología izquierdista" de la UE con respecto al matrimonio igualitario y la adopción. A su vez, se denuncia la "cristofobia" y los ataques a la memoria de Juan Pablo II, en busca de establecer procesos disciplinarios contra quienes critiquen su figura. ${ }^{17}$

El Gobierno de Ley y Justicia, en su segundo mandato, se encuentra constituido por las siguientes fuerzas políticas: Acuerdo de Jarosław Gowin (ala moderada), Solidarna Polska (ala radical), liderada por el Ministro de Justicia Zbigniew Ziobro, y PiS, facción dominante liderada por Jarosław Kaczyński. ${ }^{18}$ Queda así constituida la coalición con una fuerza moderada de Acuerdo, una fuerza radical de derecha en Solidarna Polska y un medio moderador de derecha como Ley y Justicia que cumple una función de árbitro (Bartek Pytlas, comunicación personal, 20 de mayo de 2021).

Zbigniew Ziobro, quien fue excluido de Ley y Justicia en 2011, creó Solidarna Polska, desde donde establecieron alianzas nuevamente con el Gobierno. Ziobro tiene grandes ambiciones políticas y una perspectiva más a la derecha que el propio Kaczyński, e incluso se postula como su sucesor. El partido se define en defen-

16 Programa de Ley y Justicia (2019).

17 Editorial en Newsletter de Ordo luris, del 6 de marzo de 2021. Actualmente, esta organización está inaugurando su propia universidad, Collegium Intermarium, para formar dirigentes para la derecha católica. Otro de los apoyos centrales del Gobierno es Radio Maryja, dirigida por el padre Tadeusz Rydzyk, que tiene una audiencia, principalmente, en el interior del país constituida por una población católica, mayor y conservadora, y cuenta también con el Colegio de Cultura Social y de los Medios fundado en Torún en 2001 (Michal Sutowski, comunicación personal, 3 de septiembre de 2020).

18 Jaroslaw Gowin había pertenecido a la Plataforma Cívica en el periodo 2005-2007, siendo ministro de Justicia de Donald Tusk. Había competido con Tusk dentro de su partido, pero perdió. Luego, en el Gobierno de Ley y Justicia, se le ofrece la oportunidad de desempeñar un papel importante en la política, y ejerció con su partido Acuerdo un rol para dotar de una mayoría a la coalición liderada por Ley y Justicia. Ha manifestado su oposición a ideas de Jaroslaw Kaczyński, por ejemplo, en el sistema de votos por correo que quiso implementar Kaczyński en 2020, pero prevaleció la opinión de Gowin (Paweł Śpiewak, comunicación personal, 25 de mayo de 2021; Andrzej Rychard, comunicación personal, 31 de mayo de 2021). 
sa de la "soberanía y los derechos de las familias polacas". ${ }^{19}$ Ha estado criticando al Gobierno por ser demasiado tímido ideológicamente, y representante de los sectores más conservadores de la coalición gubernamental (Szczerbiak, 2021d).

El país se encuentra atravesado por la polarización entre PiS y anti-PiS, en particular luego de las elecciones 2020 y las protestas juveniles en favor de la legalización del aborto, que mostraron el rechazo al Gobierno entre los jóvenes (Raciborski, 2020). De hecho, fueron protestas contra la sentencia del Tribunal Constitucional, controlado políticamente, que declaró la ley de aborto inconstitucional. El aborto en Polonia es casi imposible e ilegal, es una de las leyes más severas de Europa. El Gobierno estimula esta polarización entre la Polonia rural de las granjas y la Polonia de las ciudades más cercanas al intercambio cultural con otros grupos.

El Gobierno PiS ha establecido un programa de políticas sociales que ha producido una dignificación y sentido de pertenencia a la comunidad de los más pobres entre los polacos. El PiS reivindica la realización de vacaciones de los polacos por primera vez. Este proceso de dignificación de los excluidos es comparable a lo realizado en distintos países de América Latina por los populismos. En este sentido, podemos comparar con lo llevado a cabo en Argentina por el primer peronismo o en Brasil durante el varguismo o el llamado "lulismo", cuyas políticas sociales dignificaron la vida en el nordeste del país (Singer, 2009).

Ley y Justicia representa una política social generosa que tiene esa dimensión de incorporación a la ciudadanía social propia de los procesos políticos populistas, pero acompañado de una política cultural conservadora. Combina política económica de izquierda con política cultural de derecha. ${ }^{20}$

19 Sitio web de Solidarna Polska: https://solidarna.org/o-nas/

20 El fallo del Tribunal Constitucional en 2020 contra el aborto ha generado movilizaciones juveniles que podrían alterar la gramática política del país. Los estudios muestran que el número de polacos de 18 a 24 años que se identificaron con la izquierda política casi se había duplicado del 17 \% en 2019 al 30 \% en 2020 (Szczerbiak, 2021c). Las iglesias fueron uno de los principales focos de ataque de las protestas realizadas por los jóvenes. Frente a esta situación, Kaczyński declaró que había que "defender a las iglesias". En este punto, el nuevo enemigo definido por PiS son los grupos feministas y de lesbianas, gais, transexuales y bisexuales (LGTB) a los que acusa de representar la "dictadura del arcoíris". En este sentido, la familia conservadora como forma de politización tiene un potencial afectivo. Busca representar una presencia y afirmación de la comunidad frente al "neoliberalismo" (Korolczuk y Graff, 2018). Si bien los autores han señalado la adscripción importante de jóvenes a organizaciones de extrema derecha, también las protestas de 2020 a favor del aborto han sido protagonizadas por los jóvenes. Esto muestra que hay un giro a la izquierda juvenil que en los próximos años podría determinar la aparición y el éxito de propuestas de izquierda que puedan representar 


\section{La emergencia de una nueva derecha radical}

Desde 2006, se han comenzado a promover la llamadas Marchas de la Independencia, en que se congregan grupos nacionalistas con una mirada restringida a una visión homogénea de la comunidad social y política (Kotwas y Kubik, 2019). El Gobierno de Ley y Justicia, con su política cultural de derecha y anticomunista, ha originado las condiciones para la emergencia de nuevas fuerzas de derecha radical.

Una sorpresa de las elecciones parlamentarias de 2019 fue el surgimiento de una nueva fuerza a la derecha de PiS. Konfederacja obtuvo 11 asientos en el Sejm y el $6,8 \%$ de los votos (Szczerbiak, 2020). Se encuentra constituida por el Movimiento Nacional, Korwin y Korona. Distintos autores coinciden en que la base política del partido son los jóvenes, preferentemente hombres y cuentapropistas de pequeñas ciudades o del mundo rural. La propaganda del partido enfatiza en un modelo de hombre heterosexual y patriarcal. Como forma de aproximarse a grupos juveniles, se reivindican los videojuegos, la portación de armas y la virilidad, así como una concepción de la mujer ligada a la protección del hombre. El partido defiende una reducción de los impuestos y una economía de libre mercado.

Esto preocupa a Kaczyński, quien ha procurado monopolizar el espacio de la derecha (Bill y Stanley, 2020). Es la primera vez que surge una fuerza política a la derecha del Gobierno, lo que podría alterar el ecosistema político de derecha radical. El Gobierno se encuentra presionado tanto por las fuerzas juveniles de izquierda que se movilizaron en las protestas en favor del aborto como por la derecha novedosa que acusa al PiS de "traición" como Konfederacja.

Konfederacja mantiene frente a la relación entre Polonia y la UE la posición de denunciar las exigencias de la UE como "intromisión extranjera" y mostrarse como euroescépticos. También para diferenciarse de PiS, Konfederacja se opone a lo que llama "multiculturalismo" y presenta un programa económico de libre mercado.

Korwin es uno de los partidos que integran Konfederacja. Este partido defiende lo que llama la "civilización latina", "que surge del amor griego por la verdad y la belleza, los principios romanos del derecho y el imperio de la ley y la

estos segmentos (Rychard, 2021). Este fenómeno se desarrollaría, principalmente, en las mujeres jóvenes de las grandes ciudades, a diferencia de los varones jóvenes de las pequeñas ciudades o pueblos del interior. 
ética cristiana". ${ }^{21}$ El partido se encuentra liderado por la figura de Janusz Korwin Mikke. Este político conservador cultural sostiene una visión económica de libre mercado. De pasado anticomunista, tiene una visión de género patriarcal y conservadora, y eso es lo que ofrece a su electorado con declaraciones como "Las mujeres eligen a un marido más inteligente que ellas" (Sánchez, 2017). Ha participado del Parlamento Europeo desde 2014 con este voto, y ha obtenido 67928 votos en el distrito electoral de Silesia (Aleks Szczerbiak, comunicación personal, 19 de noviembre de 2020). A principios de 2015, había anunciado la formación de un nuevo partido llamado Coalición para la Renovación de la República de Libertad y Esperanza (KORWiN), y se convirtió en candidato de este partido en las elecciones presidenciales de 2015. ${ }^{22}$

Korwin combina los dos atributos que conforman el programa político de Konfederacja, con una visión cultural conservadora y una agenda de libre mercado. Sostiene que "rechaza completamente el socialismo y el principio llamado justicia social, que es contrario a los valores mencionados". ${ }^{23}$ Como vemos, hace del anticomunismo un principio de identidad.

El Movimiento Nacional (Ruch Narodowy), otro miembro de Konfederacja, tiene una visión económica de intervención estatal que defiende desde el punto de vista de la DSI, lo que inicialmente lo asemeja a Ley y Justicia. ${ }^{24}$ Sin embargo, la alianza Konfederacja sostiene una defensa del libre mercado para diferenciarse de las políticas sociales y de lo que denominan "justicia social", que son un aspecto central del gobierno de Ley y Justicia. ${ }^{25}$ Uno de los principales líderes del Movimiento Nacional es Krzysztof Bosak, quien fue candidato a presidente por Konfederacja en 2020 y defiende políticas de libre mercado. La mayor convergencia de la alianza se produce desde el punto de vista de la visión cultural conservadora sobre el orden social.

Otro grupo es el monarquista liderado por Grzegorz Braun, que lidera la Confederación de la Corona de Polonia (KKP), que, al igual que el partido KORWiN, defiende lo que llama la "restauración y preservación de la civilización latina" ${ }^{26}$ y, además, se define como un partido que reivindica "la fe, la familia y

21 Declaración del Partido Korwin: https://wolnosc.pl/deklaracja-ideowa/

22 Sitio web del Partido Korwin: https://wolnosc.pl/poslowie-korwin/

23 Declaración del Partido Korwin: https://wolnosc.pl/deklaracja-ideowa/

24 Programa de Ruch Narodowy: POLISH STRENGTH! Self-government program. Convention of the Local Government of the National Movement, Warsaw, September 1, 2018.

25 Declaración del Partido Korwin: https://wolnosc.pl/deklaracja-ideowa/

26 Estatuto de la Confederación de la Corona Polaca. 
la propiedad" ${ }^{27}$ Braun tiene pasado como periodista y militante anticomunista, como buena parte de los principales políticos de Konfederacja y PiS. Este diputado del Sejm denuncia la "hipocresía de las élites poscomunistas", que, según esta visión, han entregado el país a Washington y Bruselas. Resalta el valor del conservadurismo y el tradicionalismo. A su vez, se opone a las medidas de confinamiento decretadas por el Gobierno polaco ("De la pequeña Polonia a la gran Polonia: Entrevista a Grzegorz Braun", 2021). Esto, como hemos visto, es una actitud de Konfederacja que le permite diferenciarse del Gobierno, con la aspiración a obtener un rédito de los disconformes con las restricciones sanitarias. Tal como el partido KORWiN, Korona también defiende lo que denomina el derecho de los padres a criar a sus hijos de forma independiente de las instituciones estatales.

En definitiva, observamos la emergencia de una nueva derecha radical que pone en cuestión el monopolio en este espectro ideológico que tenía hasta entonces Ley y Justicia. Konfederacja reúne diversas expresiones radicales que se encontraban fragmentadas. Si bien culturalmente parece haber más coincidencias, económicamente aparecen diferencias, a pesar de que la alianza defiende una política de libre mercado.

\section{Conclusiones}

La consolidación en el poder de Ley y Justicia encuentra bases para su éxito en la historia de antisemitismo y el Estado monocultural católico. La derecha actual de Polonia se basa en un argumento religioso, una visión de sociedad homogénea y un discurso antiélite (y anticomunista). El Gobierno promueve un "estado de bienestar" combinado con una política cultural conservadora que niega la participación polaca en el Holocausto. El antisemitismo se encuentra fuertemente enraizado en la historia de la nación polaca moderna, lo que explica el éxito de la visión histórica complaciente que propone PiS, que colabora en la percepción de los polacos como ciudadanos patriotas y católicos, y oculta su complicidad en el pasado con los crímenes antisemitas. La combinación entre una política social incluyente para sectores populares que se perciben excluidos junto con una política cultural conservadora contra la izquierda ha sido propia también de populismos latinoamericanos del siglo pasado, como el peronismo y el varguismo.

27 Sitio web de la Confederación de la Corona Polaca: https://konfederacjakoronypolskiej.pl/ 
Estas condiciones han propiciado la emergencia de Konfederacja, que, en competencia por derecha con el Gobierno, reúne distintas expresiones de la derecha radical. Sin embargo, mientras $\mathrm{PiS}$ recurre a la carta del antisemitismo de forma muy velada y ocasionalmente en campańa electoral, Konfederacja lo hace de forma más abierta en la búsqueda por consolidar un núcleo duro de apoyo entre los votantes de derecha desencantados con la "tibieza" de PiS, al que denuncia como una falsa derecha. Además, una distinción fundamental entre Konfederacja y PiS se refiere a la economía. Mientras el partido de Gobierno se pronuncia en favor de la justicia social y contra el neoliberalismo, Konfederacja hace énfasis en el libre mercado y el valor del individualismo. Se trata de la primera formación luego de la emergencia de PiS que ha logrado reunir distintas expresiones de la derecha radical fuera del Gobierno, lo que pone en cuestión el monopolio en este espacio del espectro ideológico que tenía en forma previa Jarosław Kaczyński.

Si bien no es posible plantear una continuidad sin fisuras entre el pasado y el presente, el antisemitismo persiste en el tiempo, dada su vinculación con la constitución moderna del Estado. El antisemitismo forjó la identidad de la nación polaca como oposición a una otredad representada por los judíos, alemanes y los ucranianos, quienes fueron echados o asesinados luego de la segunda posguerra. ${ }^{28}$ Como ha señalado Pankowski, "el número de judíos en Polonia hoy es mínimo, pero el prejuicio antijudío sirve como código para una hostilidad general hacia la diversidad y la democracia (liberal) polaca" (Pankowski, 2010, p. 3). O como ha señalado Jacek Purski, "el antisemitismo es como una nota en la melodía que como político de derecha puedes tocar una y otra vez" (comunicación personal, 15 de junio de 2021).

Estas características históricas y estructurales constituyen un caldo de cultivo para el desarrollo de proyectos de derecha radical. A su vez, el marco anticomunista brindado por el rechazo al régimen luego de 1989 también es parte de los elementos dominantes. Esta situación forma parte de las condiciones que hacen exitoso el uso de un discurso nativista contra judíos e izquierdistas por parte de las fuerzas de la derecha populista radical.

Las definiciones de Casullo (2019) y Mudde $(2017,2019)$ sobre el populismo de derecha nos permiten comprender estas cuestiones. La delimitación excluyente de la comunidad en términos nativistas y religiosos de derecha tiene particu-

28 Actualmente, un $30 \%$ de los polacos señalan que no aceptarían un judío como parte de su familia (Masci, 2018). 
Ariel Goldstein
Populismo y nativismo en las derechas radicales de Polonia:

entre el pasado y el presente

larmente condiciones favorables en función de las características propias de la historia polaca.

Si bien Ley y Justicia propone un tipo de inclusión a partir de sus políticas sociales y de reivindicación nacionalista, excluye a los migrantes y activa la representación histórica de Polonia como Estado monocultural católico que se forja sobre la expulsión y el exterminio de judíos y ucranianos constituida luego de la Segunda Guerra Mundial. Las derechas radicales construyen una visión restringida y pretendidamente homogénea de la nación, que, al estar anclada en una narrativa histórica poderosa, constituye una de las bases de su éxito político.

\section{Referencias}

Applebaum, A. (2020). Twilight of democracy: The seductive lure of authoritarianism. Signal.

Altares, G. (2021, 10 de febrero). Polonia estrecha el cerco contra los historiadores del Holocausto. El País. https://elpais.com/internacional/2021-02-10/polonia-estrecha-el-cerco-contra-los-historiadores-del-holocausto.html

Bill, S. y Stanley, B. (2020). Whose Poland is it to be? PiS and the struggle between monism and pluralism. East European Politics, 36(3), 378-394. https://doi.org/10.1080/ 21599165.2020.1787161

Casullo, M. E. (2019). ¿Por qué funciona el populismo? El discurso que sabe construir explicaciones convincentes de un mundo en crisis. Siglo XXI.

Darden, K. y Grzymala-Busse, A. (2006). The great divide: Literacy, nationalism, and the communist collapse. World Politics, 59(1), 83-115. https://doi.org/10.1353/ wp.2007.0015

De la pequeña Polonia a la gran Polonia: Entrevista a Grzegorz Braun. (2021). Revista Corona, 3, 3-5.

Gdula, M. (2018). Nowy autorytaryzm. Wydawnictwo Krytyki Politycznej.

Judt, T. (2006). Postwar: A history of Europe since 1945. Penguin.

Kershaw, I. (2015). Descenso a los infiernos: Europa, 1914-1949. Crítica.

Kim, S. (2021). ... Because the homeland cannot be in opposition: Analysing the discourses of Fidesz and Law and Justice (PiS) from opposition to power. East European Politics, 37(2), 332-351. https://doi.org/10.1080/21599165.2020.1791094

Korolczuk, E. y Graff, A. (2018). Gender as "Ebola from Brussels": The anticolonial frame and the rise of illiberal populism. Signs: Journal of Women in Culture and Society, 43(4), 797-821. https://doi.org/10.1086/696691

Kotwas, M. y Kubik, J. (2019). Symbolic thickening of public culture and the rise of right-wing populism in Poland. East European Politics and Societies, 33(2), 435-471. https://doi.org/10.1177/0888325419826691

Laclau, E. (2012). La razón populista. Fondo de Cultura Económica.

Lukowski, J. y Zawadzki, H. (2019). A concise history of Poland. Cambridge University Press. 
Populismo y nativismo en las derechas radicales de Polonia:

entre el pasado y el presente

Masci, D. (2018, 28 de marzo). Most Poles accept Jews as fellow citizens and neighbors, but a minority do not. Pew Research Center. https://www.pewresearch.org/ fact-tank/2018/03/28/most-poles-accept-jews-as-fellow-citizens-and-neighborsbut-a-minority-do-not/

Mouffe, C. (2007). En torno a lo político. Fondo de Cultura Económica.

Mudde, C. (2017). An ideational approach. En C. R. Kaltwasser, P. A. Taggart, P. O. Espejo y P. Ostiguy (eds.), The Oxford handbook of populism (pp. 27-47). Oxford University Press.

Mudde, C. (2019). The far right today. John Wiley \& Sons.

Ost, D. (2018). Workers and the radical right in Poland. International Labor and Working Class History, 93, 113. https://doi.org/10.1017/S0147547917000345

Panizza, F. (2011). ¿De qué hablamos cuando hablamos de populismo? ¡Más populista será tu abuela! Revista de Ciencias Sociales de la Universidad Católica del Uruguay, 2(2), 15-37. https://ucu.edu.uy/sites/default/files/publicaciones/2018/recso_2. pdf\#page $=10$

Pankowski, R. (2010). The populist radical right in Poland: The patriots. Routledge.

Prazmowska, A. (2011). A history of Poland. Macmillan International Higher Education.

Pytlas, B. (2020). From mainstream to power: The law and justice party in Poland. En F. Decker, B. Henningsen y M. Lewandowsky (eds.), Aufstand der Außenseiter: Die Herausforderung der europäischen Politik durch den neuen Populismus. Nomos. https://www.academia.edu/download/64840382/Pytlas_Law_and_Justice_Mainstream_to_Power.pdf

Rydliński, B. (2018). Nationalism and Neo-Fascism Under Jarosław Kaczyński. En The Far Right in Government: Six case studies from across Europe. Rosa Luxemburg Stiftung. https://www.rosalux.de/en/publication/id/39160/the-far-right-in-government

Sánchez, Á. (2017, 31 de marzo). El eurodiputado Korwin-Mikke: "Las mujeres eligen a un marido más inteligente que ellas". El País. https://elpais.com/internacional/2017/03/29/mundo_global/1490794756_414867.html

Singer, A. (2009). Raízes sociais e ideológicas do lulismo. Novos estudos Cebrap, 85, 83102. https://doi.org/10.1590/S0101-33002009000300004

Szczerbiak, A. (2020, 6 de enero). What are the prospects for Poland's radical right Confederation? LSE European Politics and Policy (EUROPP). https://blogs.lse.ac.uk/ europpblog/2020/01/06/what-are-the-prospects-for-polands-radical-right-confederation/

Szczerbiak, A. (2021a, 4 de enero). How has the EU budget dispute affected the power struggle within Poland's governing camp? Notes from Poland. https://notesfrompoland.com/2021/01/04/how-has-the-eu-budget-dispute-affected-the-powerstruggle-within-polands-governing-camp/

Szczerbiak, A. (2021b, 2 de marzo). What are the prospects for Poland's TV celebrity-politician Szymon Hołownia? Notes from Poland. https://notesfrompoland. com/2021/03/02/what-are-the-prospects-for-polands-celebrity-politician-szymon-holownia/

Szczerbiak, A. (2021c, 30 abril). Can younger voters revive the Polish left? Notes from Poland. https://notesfrompoland.com/2021/04/30/can-younger-voters-revive-thepolish-left/

Walenciak, R. (2020, 9 de noviembre). Ludzie wyszli na ulice: I co dalej? Ohttps://www. tygodnikprzeglad.pl/ludzie-wyszli-ulice/

Esta última es la referencia a una entrevista a Raciborski realizada por Robert Walenciak 